\title{
Indigenous knowledge of healing among the tribes: A reference from the tribes of Kerala and Assam.
}

\author{
Rakesh Kumar ${ }^{1,2}$ \\ ${ }^{1}$ School of Humanities, National Institute of Advanced Studies, IISc Campus, Bangalore, \\ India- 56001 \\ 2 The University of Trans-Disciplinary Health Science and Technology, Bangalore, India- \\ 560064 \\ kumarrakesh9709@gmail.com
}

\section{Introduction}

Indigenous knowledge implies inter-generational passed-on understanding and skills developed by the communities. It is conventionally having a long temporal history and spatial association [1]. The vertical transfer of Indigenous knowledge from one generation to another generation doesn't imply that it is unconditionally static rather it is dynamic in a way because the knowledge modifies itself as a response to change or transition in community lifestyle and environment. However, previous writings on indigenous knowledge of healing have mostly perceived it as unconditionally static knowledge and discussed it as heritage, searching for its recognition, protecting its threatened status, and arguing for its validity against the western framework.

The indigenous approach to healing (IAH) is one such example of Indigenous knowledge and it is discussed as a kind of knowledge system which consists of a set of common values, beliefs, worldviews, experiences and practices. It comprises lived, experiential and enacted knowledge and its determinants are mind, body, spirit, ecology and socio-cultural practices. IAH is constituted locally and embedded in the practices, institutions, relations and rituals. Alike other forms of indigenous knowledge, IAH is also essentially tacit and practised orally. Though there are some unique and universal features of the IAH i.e. use of ecstasy, knowledge obtained during an altered state of consciousness (ASC), belief in spirit and supernatural world, and healing in divination [2]. It forbids IAH to comply with the bases and framework of the empiricist model i.e. knowledge can be only acquired through physical senses, acknowledged through virtual experiences as valid and objective, obtained through as a result of the practice of method and it doesn't imply belief (which is the core of IAH) [3]. Therefore, there has been always a general disagreement regarding the placement of IAH within the domain of knowledge systems and religion. The practitioner of the empiricist model and non-traditional healer (in anthropological categorisation- professional healing practitioner) categorised IAH as an animistic form of religion rather than a knowledge system.

Unfortunately, most of the writings (on IAH) were also baffled by the misconception and false labelling of IAH (by empiricist framework) and set the motion rolling for validating IAH through the western scientific or empiricist framework. In the process of validating IAH, scholars have consciously or unconsciously kept a distance from the metaphysics of indigenous knowledge and only focused on the material used in indigenous healing. For example, the existing writing on IAH mostly dealt with the scientific investigation of extensively diverse but insufficiently documented knowledge of ethnomedicinal plants, zoological secondary 
compounds, minerals and other physical substances. Scarcely these kinds of studies involved in the philosophical inquire of indigenous knowledge of healing.

The obvious reasons that seem accountable to choose material over epistemology and metaphysics of IAH could be the contested notion of healing, changing concepts of health or disease, the inclusion of supernatural agents, use of ritual in healing, diversity of worldviews and belief system in the inquiry of health. The other intention that appears logical for excluding metaphysics as source or data is that it is at odds with the materialist and empiricist framework. Therefore, the exclusion of metaphysical data that question the sustainability of a model appears an easier way rather than to include those and involve in deep inquiry and change the assumption involved in modelling. At the same time, another criticism that IAH often encounters is the non-replicability of process. A good response to this criticism comes from the debate of subjectivity and historical continuity of the tradition that addresses the flaws in the criticism (detail discussion may require a separate article) but before that few important questions are needed to be answered in context of the present article. Is there an indigenous approach to health and diseases? Is there any philosophical concept of indigenous healing?

In this article, the above-mentioned questions will be analysed with the help of a case study of the selected indigenous tribal communities of Kerala and Assam. In due course, the researcher will also try to discuss different models of IAH and how the model of IAH is different from other existing model of health and healing.

\section{What is the purpose of healing?}

As discussed above there is no single definition of healing. However, etymologically healing comes from the old English word hèlan meaning 'wholeness' [4]. But again wholeness in what sense? Is it a process or an experience? Is it functional or desired? Or is it an act of transcendence or transformation? Each discipline of knowledge define healing differently, therefore, the concept remained confusing and inexact. The western healthcare discipline inspired by naturalist views describes healing in functional terms as an activity of the profession of medicine [5]. However, the proponents of holistic views delineate it in experiential terms- transcending suffering and transforming it into wholeness [6]. If this is so, Can there be a single definition of healing? Is there any philosophy of healing? Will an operational definition of healing suffice the explanation of the mechanism?

To answer these questions, it is important to analyse the various attributes which are connected to the concept of healing. Healing in medicine has been understood as a restoration of health from an unbalanced, diseased, damaged or unvitalized organism [7]. According to this definition, healing is a relative term and can be understood from bodily or biological terms. Whereas, Firth and et al. (2015) has identified four attributes of healing after performing concept analysis and extensive literature reviews on healing. According to him, 'Healing is a holistic transformative process; it is personal; it is innate or naturally occurring; it is multidimensional; and it involves repair and recovery of mind, body, and spirit' [8]. But in all cases, the prime focus has been placed on the concept of repair/recovery. This suggests that healing is not a spontaneous phenomenon rather it is a necessity based i.e. it requires in case of a deviation from one's earlier state of being (positive sense). The deviation can occur at levels i.e. micro (cellular) to macro (nation or global) [7] [8]. In the philosophical term, the deviation can crop up at the level of body, mind, self/spirit [8]. Therefore, healing can have multiple dimensions like physical, mental, emotional, spiritual, social, communal, environmental etc. It 
means healing is a dependent phenomenon and hard to explain in isolation. The diagram (fig1) concisely comprehend the complexities of healing.

But the fundamental question regarding healing is still unanswered. Is healing an intervention or a process or an outcome or all three at times? As it is discussed above, healing is a dependent phenomenon that is often described in the context of another complementary term i.e. health. Therefore, to contextualize the debate of healing it becomes essential to analyse the philosophy of health, its dimensions and determinants. It is also important to note here that the root word for health and healing are the same and they are both derived from the old English word heel meaning wholeness.

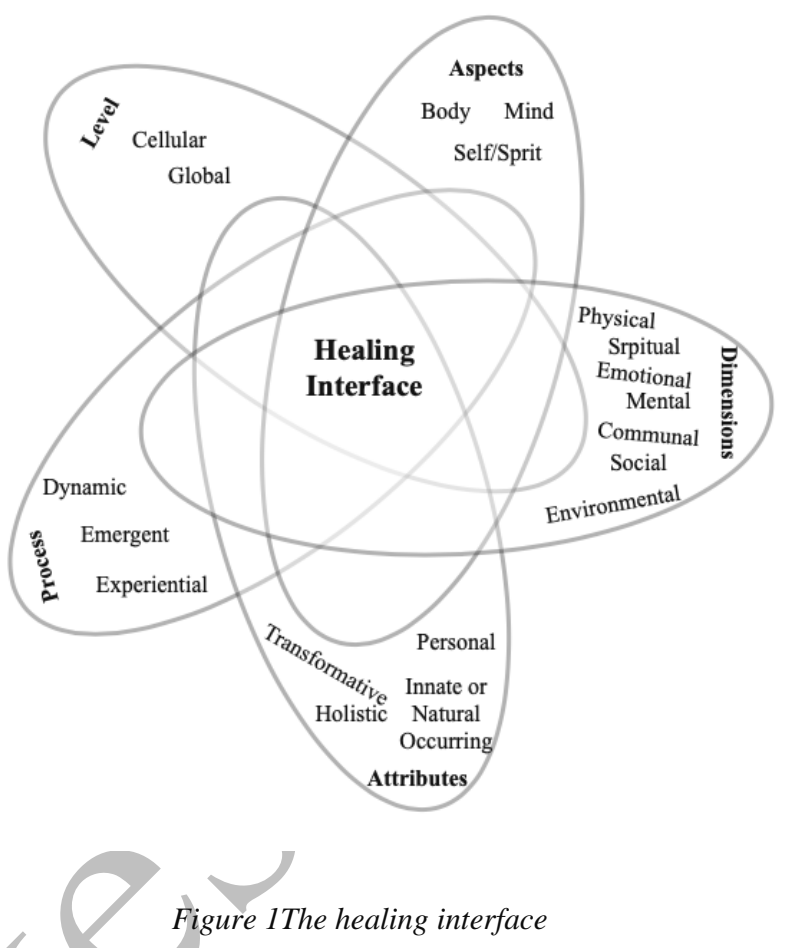

Alike healing, the concept of health is also constantly changing. The bio-medicinal concept views health as 'absence of disease' [9], Ecological concept implies health in terms of adaptation [10] and for the Psychosocial model - health is both biological and social phenomena [11]. Whereas, the proponents of the Naturalist view describe health in terms of physical, natural, biological or physiological processes that can be measured and are observable with modern technology [12]. For example, Boorse' Bio-statistical Theory of Health (BST) posit that a 'person is healthy if and only if, all-natural organ function normally given a statistically normal condition' [12]. Boorse' definition of normal for a functioning organ is based on 'species design', in which each organ can be measured separately in statistical terms [9]. But what is problematic with this definition is that it evaluate parts not the whole body as a unified and connected system. For, BST all abnormal functions are diseases. But intuitively it's not the case always. For example, Autistic people. In this case some organs are super functioning (i.e. functioning at a higher level than the normal species design). In autistic case organ certainly does not function following normal species design. Can it be considered unhealthy? The question remains unanswered.

Whereas, the holistic philosophy imply health as a value-laden phenomenon e.g. vital goals, meaning and purpose [9]. For example, the Holistic Theory of Health (HTH) posit that a person 
is healthy if and only if (given standard circumstances) he/she can attain their vital goals [13]. In this definition, health is characterised positively in equilibrium (not in terms of biological homeostatic) between capabilities and goal [9]. Though HTH does not characterise health in statistical terms at the same time, it also does not characterise in a non-biological term. For example, according to HTH diseases (a state of anatomical, physiological and psychological that tends to restrict capabilities) affect the individual's capabilities. The other problem with HTH is the definition of vital goals which is 'a state of affairs which is either a component of or otherwise necessary for the person's living a minimally decent life' [14]. For example, there cannot be a universal minimal decent life because it is socio-economic contextual. The decent life of a hunter-gatherer would be entirely different from a sedentary community. Even in a similar socio-economic context, the minimal decent life for a moderate-income individual will be different from a billionaire. The non-consideration of the definition of vital goals also doesn't make any difference. For example, what if an individual sets his goal at a very low level. He/she can have many diseases and malfunction but he is in a position where he can hardly become unhealthy according to the definition of HTH.

However, there are various hybrid and integrative models which have tried to synthesize the concept of health and develop a philosophy of health. One of them is developed by Saad and Prochaska (2020). According to this model 'heath is a state of maintainable-ease of functioning and a disease is a state of prolonged dysfunction that prevents ease' [9]. In simple words, the state of dis-ease in being can be considered unhealthy. It provides a systemic view of health and attempted to integrate the biological function, behaviour function and social function of an individual or a system. According to this philosophy, health can be observed at multiple levels e.g. at the level of cell, self, and society. Therefore, the determinants of health can be anything both from the internal and external world of the individual. For example, biological condition, behavioural condition, socio-cultural condition, environment, socio-economic condition, services, age, gender, food, education etc.

Despite these many models, several questions remain unanswered. For example, Is health a property concept or relation concept? Is health evaluative or descriptive concept? Is it a state of body or mind? What kind of logical opposition exists between health and diseases? Are disease and health contrary concepts or complementary concepts? Hence, to explore these questions, the article has shifted its focus towards IAH. How do indigenous people perceive health and disease? What is the epistemology of IAH? And for the case study, the researcher has selected indigenous communities of Assam and Kerala.

\section{Case Study}

India is the home of 550 Indigenous/tribal communities. Out of 550 of India, Kerala is the home for 35 tribal communities and Assam is for 29 tribal communities. It is important to mention here that the term Indigenous has been loosely used for tribal communities or ethnic groups to avoid the perplexity of terms. The other reason for choosing the term 'indigenous' is that it is widely accepted and being used by the United Nations. Scholars have outlined a few common characteristics of the Indigenous people which may and may not apply to all. Though these characteristics make the term indigenous an ambivalent concept because according to these characteristics aren't we all are indigenous to some land where we have born? The characteristics are as follows:

- The historical continuity of inhabitation over a specific geographical space or territory

- Self-identification as with a distinct name and exhibit a strong sense of belonging or identity which is accepted by both the individual as well as the community 
- Having a discrete belief system, language, religion and socio-cultural practices

- Politically autonomous (that is the tendency to manage their affair separate from the government) and economically self-sufficient

Out of 35 tribal/indigenous communities four communities namely Cholanaickan (C), Kattunaickan(K), Kattupaniyan(P), and Aranadan (A) are selected from Nilambur valley, Kerala and two communities namely Tai-Ahom and Missing from Assam. The communities from Kerala were and are (to a substantial level) nomadic and foraging communities. Their subsistence strategy includes hunting, food gathering, and fishing. However, the communities from Assam are sedentary. All the selected communities have their own tradition of healing, which is non-codified and practices orally. The data informing this article comes from both primary and secondary data sources. The primary data collection includes the sources (I) qualitative data in the form of in-depth interviews (IDIs), and focus groups discussion (FGDs) and (II) ethnographic data were collected in the form of quasi-participant observation. Before data collection, informed consent was taken from the respondents and participants.

\section{The indigenous concept of health and healing: Based on references from selected tribal communities}

Existing literature on indigenous healing tradition equates the indigenous model of health (IMH) with the holistic approach in which to ensue health body, mind, spirit and emotion must be in the state of balance and harmony. Any imbalance or disruption among these can be considered an illness. The idea behind this definition of health is the philosophy of wholeness or interrelatedness i.e. everything having a life is interconnected and affects each other, which indigenous communities are subscribed to. This is a very broad definition of health that is interesting and inclusive. But according to this definition, nobody will be in the position of health because there will be always some or the other kind of disharmony between the internal factor and external factor. After all, the system is dynamic rather than static. For example, Every day our ecology (of which we humans are a vital component) is changing and resulting in some or other form of both internal and external imbalance. Can this imbalance call as an illness? If this is so, Is everyone ill? This is also important to note that here by no means the researcher has set out to devalue or slag the above-mentioned model/definition of indigenous health. But on the other hand, the attempt has been made to look for an alternative model of health with the help of indigenous wisdom (if it is there).

Health among indigenous communities has three indicators- (a) productivity, (b) causal contribution to the larger goal of society and (c) expected functioning behaviours/abilities. These three indicators have been deduced from the interactions with selected indigenous communities of Kerala and Assam. Here productivity implies the efficiency in the production of any kind and expected functioning behaviours/abilities referred to functioning according to pre-determined conditions. In that sense, health becomes a conditional property of being. For example, as an answer to the question of 'how do you know that a child/someone is sick or unhealthy in case he/she doesn't tell himself/herself?' ', the common responses from the chosen communities of Nilambur valley, Kerala were as follows:

\footnotetext{
1 This was a common question asked during the IDIs and FGDs recording among the selected communities (A, C, K, \& P) of Nilambur valley, Kerala. The IDIs \& FGDs were recorded between December 2019 and March 2020.
} 
"......the mother can understand the mood or condition of the child. It's a common phenomenon. Once they get the disease they use to stop playing with other children, eating less, always crying, etc. ......"2.

"........the older generation people have knee and back pain issues so they are no longer able to come with us in the forest..."3
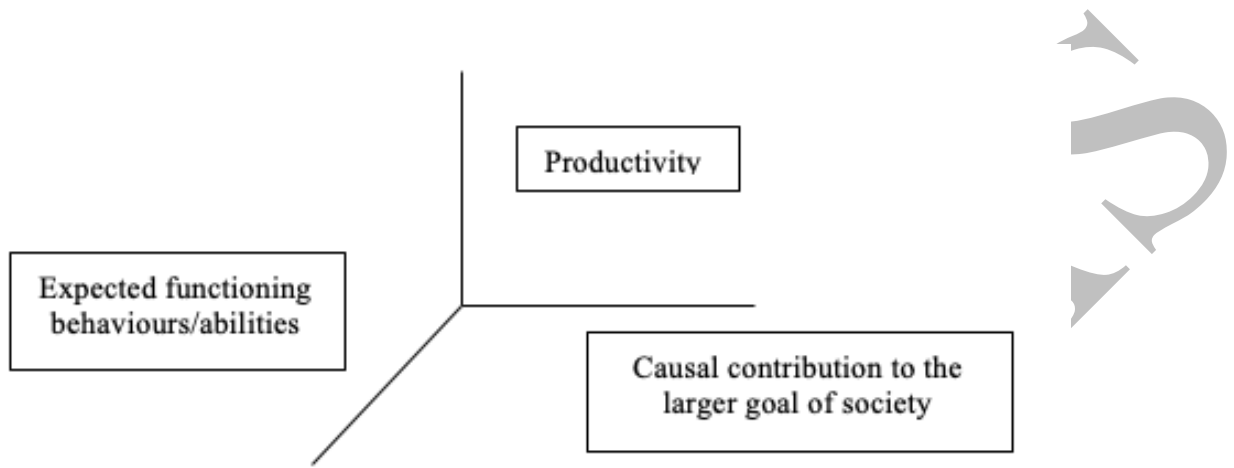

Figure 2 Indicators of health in IMH

But this does not mean health is restricted to only these three indicators of IMH. It has various other dimensions self, mind, body, emotion, culture, language, and environment. For example, health malfunctioning among the indigenous community can arise due to identity deficits, loss of language, changing relationships with the land, political unrest etc. But the above mentioned three indicators makes IMH inclusive and self-explanatory compare to Naturalistic Model (BST) [12], Holistic Model (HTH) [13] and Integrative Model (BST \& HTH) [9] in term of explaining the complex situation. For example, pregnancy. According to the models (BST, HTH and Integrative Model of BST \& HTH) discussed above, the period of pregnancy should be considered unhealthy because during pregnancy women are neither normal by species design nor functional at maintainable ease. But despite that hardly the period of pregnancy is consider has unhealthy while in actual sense it's quite opposite i.e. not getting pregnant is considered as unhealthy. Whereas, on the other hand, the indigenous model of health doesn't consider the period of pregnancy unhealthy. It is because IMH explains pregnancy as productive work, a contribution to the larger social goal i.e. producing progeny by following certain predefined expected functioning abilities. This model is not only inclusive of the disease process but also inclusive of injuries and accidents. IMH considers injuries and accidents as an event rather than a process. However, the IHM model excludes many of the bio-statistical diseases/illnesses and doesn't categorize them under health problems e.g. skin diseases, respiratory, gastrointestinal diseases, night blindness, etc. These are excluded from the categories of diseases because these do not affect the above-discussed indicators of health.

Nevertheless, the causal explanation of diseases or illnesses of IMH is also disparate from the other existing model. IMH explain disease with the help of native logic that includes natural

\footnotetext{
2 This was the common response from all the selected four selected communities (A, C, K, P) of Nilambur valley, Kerala. But this particular extract is taken from the reply of a woman of A-community from Kattupara colony during FGD in February 2020.

3 This quotation is from the K-community from Kulbalpara colony. This was a response to the question cited above. It was recorded during FGD in January 2020.
} 
(like heat and cold) to supernatural (god's anger, spits, and evil-eye) explanation of diseases. Whereas IAH includes cultural relative experiences, beliefs, worldviews, values, imaginations, and knowledge about plants, animals, and landscape.

For example,

"........Do you know the scientific way of this? Most people believe in the doctor's treatment nowadays. If a person believes in God, he gets relief. If a person is a great devotee of God, he gets relief from disease. We do the same thing through mantras and exploit their beliefs then give medicines. If a person feels peace and happiness that helps to cure half of the diseases. A psychologist brings the patient's mind back first. Then treat them."

As it is discussed above that IAH place emphasis on the use of ecstasy, belief in spirit and supernatural world, ASC, and healing in divination. However, the meaning of healing in indigenous wisdom is somewhat similar to Kok (2016) definition of healing i.e. "restoring the person to the life context". This understanding of healing is slightly different from the Naturalist and another model in which healing stands for repair or recovery. The main difference in the perception of healing between the indigenous model and other models is that the former model discussed healing in an existential context whereas the latter defines it as a fixing or improvement process.

Nonetheless, it is important to explore that what are the other dimensions and determinants of IAH? Along with emphasis over the above mentioned key aspects, IAH involves (a) knowledge about the plants and their secondary compounds, animals and their by-products, water, soil, and other minerals, (b) special sets of skills like massage (c) healing with the help of mantras, magico-medicinal practices, and (d) removing of a malevolent external agent, and amelioration by veneration or sacrifice to the specific god/ancestral power. For example, among the Cholanaickan community of Kerala IAH includes special sets of skills are like massagePidichukodukkal and Oddanereyaakal and use of mantras, magico-medicinal practices Orissadu, Koriorissadu, Daivaadal. This is not limited to one particular community, each selected communities have some form of these sets of skill and ritual/faith healing practices. It is important to mention that here faith or ritual healing is loosely used for IAH in which the use of ecstasy, mantras, magico-medicinal practices and ASC are involved.

Other than the concept and approach to health, disease and healing, IAH is also structurally different from the modern scientific discipline of medicine in the following ways. In the modern scientific approach, health is a subject of professional scrutinization and there exists a separate functional medical institution and professionals for the evaluation of health and perform healing. Whereas, for IAH the structure of healing institutions is fundamentally different. Among Indigenous communities, there hardly exists any separate functional medical institution or professional. For example, among the selected tribal communities of Kerala, there is no full time or professional healer. Even if there is a healer (professional sense), his/her role as a healer is not fixed, unlike modern doctors. He/she performs various public roles like the head of the community, judge, representative, priest etc. Whereas on the other hand if there is

\footnotetext{
${ }^{4} \mathrm{~A}$ healer from C-community responded (during IDIs) when the researcher asked the question "what is the reason for these diseases? Do you believe in the supernatural power behind this?"
} 
no tradition of professional healer or no separate institution, health is both individual and community affair and the act of healing involves collective knowledge that is stored in the cultural cloud and ecology (in the form of an image beyond nomenclature).

How do IAH function in the absence of separate institutions or professionals? In the absence of separate institutions or professionals process of healing involves self-medication/selfhealing processes by altering/modifying the diet, application of ecological knowledge, and in some cases getting assistance from the imitate family members, neighbours, or kinship members. This assistance includes sharing of medicinal knowledge, sharing of medicinal material, use of special sets of skills, caregiving etc. If the disease/ illness is serious, unfamiliar, life-threatening, or produced by systemic diseases in that case expertise of a professional healer from the outside community are taken into the consideration. It makes the act of healing more public than private because it involves sharing of efforts, experiences, knowledge, exploration of an external agent of causation (i.e. supernatural) as well as the responsibility of outcomes of healing.

Considering the diversity of IAH, the effort has been made to summarize IAH with the help of two models (the model in sense of categorization).

(A) Recursive Model: In this model, the researcher has categorized the practical experiencebased perception of health, and diseases/illness. The philosophy of this model is founded on everyday experience and an encounter with ecology, plant, and non-human organism. It follows the native logic; moulded-in ethos and philosophy of life; uses experiential knowledge deposited in the cultural cloud; incorporates family or immediate small group; and involve selfhealing or self-medicating process. This model reflects healing of diseases as a public affair and comprises both the natural as well as a supernatural intervention at both ends (i.e. for the perception of diseases/illness or construction of healing mechanism).

(B) Idiosyncratic Model: Under this model, a more severe, unfamiliar, concealed, and lifethreatening kind of disease/illness has been characterized. This model explores casual references for the diseases/illness into nature as well as cultural belief systems. It consists of environmental factors as well as the advent of supernatural beings into the life dimensions of people, for example, ancestral curses, actions of spirits, and negative actions. In this model, the scope of the caregiver is not limited to the experiential knowledge of the individual, immediate group, or co-family members but it includes the expertise of professional healers from distant group members. Along with the healing modes of the 'recursive model,' it also includes the elaborated rituals, venerations, invocations, and sacrifices in the healing process. The logical and empirical explanation in this model of health problems and healing methods incorporate historical, spiritual, personal reasoning, and agencies outside the group.

From the above-discussed model, it is clear that IAH has two dimensions of healing i.e. the dimension of individual healing and the dimension of collective healing. The dimension of individual healing involves personal health and healing of mind and body through spiritual and emotional healing (as described in 'recursive model of healing') while the dimension of collective healing comprises cultural wellbeing along with individual wellbeing. Both the healing model has two-part (a) diagnosis and (b) treatment/ application of therapeutic remedies. The diagnosis of diseases/illness incorporates the close observation of (long and short term) behaviour, normal functional abilities, touch, and change in colour (of the body or body waste), temperature and other psychosomatic changes. Whereas, treatment/ application of therapeutic remedies include caregiving (both from immediate or distant community members), use of 
herbal medicine, animal products, rituals, ecstasy, mantras and ACS. It is important to note that the method of IAH is neither uniform nor universal and it may vary greatly from community to community and healer to healer.

\section{Is there any philosophical concept of indigenous healing?}

To comprehend and apricates IAH, it is essential to acknowledge indigenous philosophy (IP) first. From the above discussion, it is clear that IP has three intricating worlds i.e. physical world (land, sky and living organism), the human world (knowledge and approach to people, family rituals and capacity to change) and the sacred world (metaphysical and spiritual) [15]. This implies that the core idea of IP is interconnectedness or wholeness. Though there can be seen diversity in worldviews and beliefs system (among various indigenous communities) but the triangulation of the three worlds is the core and universal feature of indigenous philosophy.

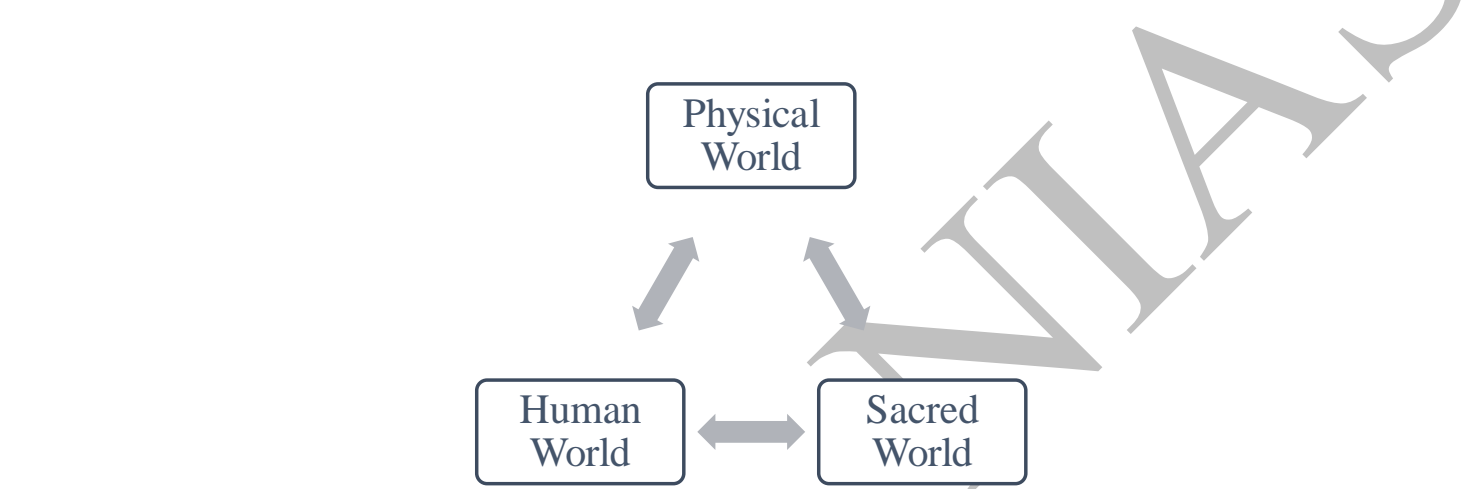

Figure 3 The three worlds of indigenous philosophy. The model of three interreacting worlds has been adapted from the Foley, D. (2003) article entitled 'Indigenous epistemology and Indigenous standpoint theory'

It is also explicit from the discussion that IAH involves various dimensions of life (i.e. intellectual, physical, emotional and spiritual) and establishes a transpersonal relation also with the non-human organism (like- animals, plants, spirits, ghosts, ancestors and gods) for the perception of health and practice of healing. In a nutshell, IAH is a dynamic mixture of tradition and present intervention which comprises native belief systems, empirical apprehension and worldviews. But it is important to note here that the physicalist and materialist philosophies (to which modern science are subscribed) negate the third component i.e. the sacred world by inscribing it unreal [16]. In a simple sense, it negated both the knowledge and experience gained from the sacred world or in ASC, which is the core of IAH.

It impels the root of IP embedded in the debate of reality and the perpetual diversity of culture. The reality in philosophy is a contested concept. Different schools of philosophies have interpreted reality differently. The physicalist approach (to which the materialist science are subscribed) finds reality only in physical objects whereas, on the other hand for the idealist, the reality is fundamentally immaterial (of which IAH have resemblance) [17]. To contextualize the debate of reality will need a separate article. Therefore, the attempt has been made to explain indigenous philosophy without delving deep into the debate of nature and the existence of reality.

To problematize the debate of reality in an indigenous context, it is important to understand to binary concept devised by Laughlin (2013) i.e. monophasic culture and polyphasic culture. According to him, monophasic culture (modern materialist western cultures) defy the alternative states like dreams and trans-state as unreal and only 'focus on adaptational 
interactions with the external physical world' [16]. Whereas the polyphasic cultures (like indigenous culture) value the experience had in ASC as a different aspect of reality rather unreal [16]. In that sence, reality in indigenous knowledge is not understood 'on the basis of a linear conception of cause and effect, but rather as a world made up of constantly forming multidimensional cycles in which all elements are part of an entangled and complex web of interactions' [18].

The epistemology of indigenous knowledge is not only diverse from the western knowledge framework in terms of monophasic culture and polyphasic culture but it is fundamentality different in multiple terms. From the above discussion, it is clear that indigenous knowledge contains both empirical knowledge and worldviews, therefore, it is not possible to reduce it to only practical knowledge that is only based on experience. The fundamental differences between indigenous and western knowledge systems are as follows. Western knowledge favours analytical and reductionist approaches in contrast to intuitive and holistic views of indigenous knowledge. On one hand, western knowledge is objective, materialist, positivist and quantitative, whereas on the other hand the indigenous knowledge is subjective, quantitative, spiritual and doesn't make a distinction between empirical and sacred [18].

In a nutshell, it can be said that the ethno-philosophy of IAH is based on a 'polyphasic culture' of transpersonal experiences and meaning-making process which is fundamentally different (in the above-mentioned terms) from the philosophy of the western model of healing.

\section{Discussion and conclusion}

From the above discussion, it is clear that healing has got less philosophical attention than health and the debate of healing revolves around the three concepts i.e. heath, disease and normality. Heath is defined in two terms mostly as an absence of disease or presence of something (a positive state). It can be also deduced from the above discussion that the naturalistic view perceive health either through empirical judgement about human physiology or normative judgement about human behaviour or wellbeing and healing implies an act of repair or recovery. Whereas the IAH coded into household practices, culturally modelled beliefs and worldviews and the knowledge system is entrenched in historical context, deposited in environmental context and encompasses both the empirical (plant and animal) and unempirical (faith healings) domains. Moreover, the religious background, cultural belief and cognitive mechanism are an essential part of both the perception of health and healing mechanism. IAH' reasoning and logic are devised by the everyday experience and daily practices in which individual behaviour is cyclic and non-reflective. The ritual and mantras of IAH evoke the transpersonal experience and integrate the altered state of an individual's consciousness with the social identity and cultural experiences.

But what is the utilization of indigenous knowledge of healing? To answer this question is it important to understand another question i.e. why are indigenous knowledge of healing excluded from the professional health practices? Since the $17^{\text {th }}$ century (with epistemological revolution) the western medicine had reduced itself into a mere function term by fixing to extreme empiricist model and discarded IAH because of the use of ecstasy, supernatural element and knowledge association with ASC [19]. It has resulted in the growing dissatisfaction in western medicine because of its fragmented view, loss of the core philosophy of healing, mechanical process, non-consideration of an individual's identity, culture and worldviews, curative approach rather than preventive approach and inefficiency to many health problems like cancer. Whereas on the other hand, IAH functions organically and it helps to 
bring a positive change and find meaning in life. In that sense, IAH connects the self with wholeness, in which western medicine is constantly failing [2]. Moreover, in IAH the insufficiently explored knowledge of ethnomedicinal plants and ethnozoological compounds also provides a tremendous opportunity for the search for a new medicine for deadly diseases.

But the technology-based development, industrial modernity, displacement-based development and capitalisation of nature have collaboratively disrupting the model of IAH. It is because they have caused the fundamental changes in indigenous people' relationship with the environment, self-other interaction and interdependence between self and sociality. It has ultimately resulted in the shifting of meaning, experience and disposition of beliefs, which is the core of IAH . Therefore, it is important to not only comprehend and apricates the epistemology and metaphysics of IAH but to preserve and protect the indigenous community and their knowledge system in their primary context for the future generation.

Bibliography

[1] M. Bruchac, "Indigenous Knowledge and Traditional Knowledge," in Encyclopedia of Global Archaeology, New York, Springer, 2014, pp. 3814-3824.

[2] R. Struthers, V. Eschiti and B. Patchell , "Traditional indigenous healing: Part I.," Complementary therapies in Nursing and Midwifery, 10(3), pp. 141-149, 2004.

[3] A. Kenny, Rationalism, Empiricism and Idealism, Oxford: Oxford University Press, 1986.

[4] J. F. Quinn, Quinn JF., New York: NLN Press, 1997.

[5] P. Starr, The Social Transformation of American Medicine, New York: Basic Books, 1982.

[6] D. McElliott, "Healing: the journey from concept to nursing practice," Journal of Holistic Nursing, vol. 28, no. 4, pp. 251-258, 2010.

[7] T. R. Egnew, "The meaning of healing: transcending suffering," The Annals of Family Medicine, vol. 3, no. 3, pp. 255-262, 2005.

[8] K. Firth, K. Smith, B. Sakallaris, D. M. Bellanti, C. Crawford and K. C. Avant, "Healing, a concept analysis," Global advances in health and medicine, vol. 4, no. 6, pp. 44-50, 2015.

[9] J. M. Saad and J. O. Prochaska, "A philosophy of health: life as reality, health as a universal value," Palgrave communications, vol. 6, no. 1, pp. 1-11, 2020.

[10] H. Mallee, "The evolution of health as an ecological concept," Current Opinion in Environmental Sustainability, vol. 25, pp. 28-32, 2017.

[11] P. Martikainen, M. Bartley and E. Lahelma, "Psychosocial determinants of health in social epidemiology," International Journal of Epidemiology, vol. 31, no. 6, p. 10911093, 2002.

[12] C. Boorse, A rebuttal on health. In: What is disease?, Totowa: Humana Press, 1997.

[13] L. Nordenfelt, "The concepts of health and illness revisited," Medicine, Health Care and Philosophy, vol. 10, no. 1, pp. 5-10, 2007.

[14] S. Venkatapuram, "Health, vital goals, and central human capabilities," Bioethics, vol. 27, no. 5, pp. 271-279, 2013. 
[15] D. Foley , "Indigenous epistemology and Indigenous standpoint theory," Social alternatives, vol. 22, no. 1, pp. 44-52, 3 .

[16] C. D. Laughlin, "The Ethno-Epistemology of Transpersonal Experience: The View from Transpersonal Anthropology," International Journal of Transpersonal Studies, vol. 32, no. 1, pp. 42-50, 2013.

[17] D. G. Ritchie, "What is Reality?," The Philosophical Review , vol. 1, no. 3, pp. 265283, 1892.

[18] F. Mazzocchi, "Western science and traditional knowledge: Despite their variations, different forms of knowledge can learn from each othe," EMBO reports, vol. 7, no. 5, pp. 463-466, 2006.

[19] L. Nordenfelt, "Health and disease: two philosophical perspective," ournal of Epidemiology and Community Health, vol. 40, no. 4, pp. 281-284, 1986.

[20] J. Kok, New Perspectives on Healing, Restoration and Reconciliation in John's Gospel, Leiden: Brill Academic Publication, 2016. 